\title{
Collisionless mirror mode trapping
}

\author{
R. A. Treumann ${ }^{1,2}$ and W. Baumjohann \\ 'Max-Planck-Institut for extraterrestrial Physics, 85741 Garching, Germany \\ IInternational Space Science Institute, 3012 Bern, Switzerland
}

Received: 29 October 1999 - Revised: 20 May 2000 - Accepted: 30 June 2000

\begin{abstract}
We investigate trapping of mirror modes in a magnetic slab. This model is a simplification of a real situation in front of the magnetopause where miror waves may become trapped in a region close to the magnetopause for tangential discontinuity conditions and an unidentified (hypothetical) boundary deeper in the sheath which we, for simplicity, assume to be another tangential discontinuity. Such magnetic slabs may trap mirror modes selecting a particular perpendicular wave lengths which follows from a quantization condition on the perpendicular wavenumber.
\end{abstract}

\section{Introduction}

For about four decades since the pioneering work of (Chandrasekhar et al., 1958) the mirror mode has been known as one of the two basic macro-instabilities in plasma containing an anisotropic thermal pressure $p_{\perp} \neq p_{\|}$, the other and complementary instability being the celebrated firehose mode. The subscripts $\perp, \mid\}$ in the above expression refer to the directions perpendicular and parallel to the local (average) magnetic field $B$. Both their physical mechanisms have been clarified first by (Rudakov and Sagdeev, 1961). The firehose mode arises under the condition that $\beta_{\|}-\beta_{\perp}>2$, where $\beta=$ $2 \mu_{0} p / B^{2}$ is the ratio of thermal to magnetic energy density. Because the parallel pressure $p_{\|}=n m\left\langle v_{\|}^{2}\right\rangle / 2$ is proportional to the (ensemble) averaged parallel energy corresponding to the centrifugal force on a bent magnetic field line, its mechanism is easily understood to result from the excess in centrifugal energy exerted on the field over its restoring force energy. This is a branch of the ordinary Alfvén wave which may be driven either by the parallel excess in ion or also electron pressure anisotropies (Hollweg and Völk, 1970, 1971).

In contrast, the mirror mode does not present such an easy interpretation (Southwood and Kivelson, 1993). The fluid theory of the mirror instability has been given by e. g. Barnes (1966) and Tajimi (1967). In the fluid picture in anisotropic

Correspondence to: R. A. Treumann, MPE, P.O.Box 1312 plasma $\left(p_{\perp}>p_{\|}\right)$any disturbance in the perpendicular pressure is in anti-phase with the corresponding disturbance of the magnetic field. For large enough anisotropies the plasma responds by pressure imbalance to changes in the magnetic pressure leading to instability. However, the fluid concept does not give the correct growth rate because it does not take into account the effect of the resonant particles with parallel velocities $k_{\|}\left|v_{\|}\right|<|\gamma|$ as has been shown by Southwood and Kivelson (1993). The particle distribution consists of two components: those trapped resonant particles and untrapped particles at higher parallel speed. Kivelson and Southwood (1996) analyzed the nonlinear evolution of the mirror mode. They have shown that in the nonlinear state increasingly more particles become trapped in the mirror mode. The mirror mode saturates when their contribution becomes more important than that of the resonant component. It should also be noted that observation of Lion roars (Tsurutani et al., 1982) related to magnetosheath mirror modes provides evidence of a trapped electron component (Baumjohann et al., 1999) in the mirror modes.

There are plenty of observations of the mirror mode in the environment of Earth, Jupiter, and comets (Tsurutani et al., 1982, 1984, 1992; Hubert et al., 1989; Lacombe et al., 1992; Glassmeier et al., 1993; Fazakerley and Southwood, 1994a,b; Fazakerley et al., 1995; Hill et al., 1995; Lacombe and Belmont, 1995; Lucek et al., 1999a, 1999b; Tátrallyay and Erdös, 1999). A favored place of the mirror mode is the magnetosheath. In addition field line draping around the magnetopause provides another free energy source for the mirror instability close to the magnetopause. Hill et al. (1995) have shown that the instability criterion for the mirror instability is marginally satisfied from about half way to the bow shock up to the magnetopause. For a timely review on the most abundant observations obtained in the magnetosheath and their interpretation the reader is referred to Schwartz et al. (1996). Recent measurements at the dawn side of the magnetopause and AMPTE IRM measurements have demonstrated that the mirror mode reaches relative amplitudes of $|\delta B / B| \simeq$ 1 while the direction of the magnetic field changes only slightly 
(see Lucek et al., 1999a,b). Early numerical simulations (Price et al., 1986) have confirmed this feature. The mode is nearly purely compressive and its wavelength along the magnetic field is much larger than the perpendicular wavelength, $k_{\|}<$ $k_{\perp}$. The observation of such large amplitudes in $|\mathrm{B}|$ suggest that the mirror mode is rarely observed in its linear state though Lacombe et al. (1992) reported some cases of linear mirror instability.

\section{Review of Linear Theory}

Given a positive pressure anisotropy $A_{j}=p_{j \perp} / p_{j \|}-1>0$ of ion species $j$ and assuming that the electrons are isotropic and cold, it is not difficult to derive the condition for instability of infinitesimally small amplitude mirror waves either from the double adiabatic MHD equations or from the Vlasov equation in the extremely low frequency limit for nearly perpendicular propagation (e.g. Hasegawa, 1969, 1975). In the fluid picture the mirror instability arises from the perpendicular pressure and magnetic field variations being in antiphase. At strictly perpendicular propagation the instability does not exist. Southwood and Kivelson (1993) have shown that a physical understanding of the mirror mode relies on the realization of the central role of resonant particles with parallel velocities $v_{\|} \sim 0$. Inclusion of finite electron temperatures introduces additional electron pressure and parallel electric field effects which affect the dispersion relation (e.g. Pantellini and Schwartz, 1995; Pokhotelov et al., 1999).

\subsection{Temporal Growth}

Following Hasegawa (1975), the kinetic dispersion relation of the combined mirror and firehose instabilities in a Maxwellian ion plasma in the presence of a cold electron background reads

$$
\begin{aligned}
\frac{k^{2} v_{A}^{2}}{\omega^{2}} & =1-\frac{k_{\|}^{2} v_{A}^{2}}{2 \omega^{2}} \sum_{j}\left\{\frac{\beta_{j \perp}}{2} \frac{A_{j}}{A_{j}+1}\right. \\
& \left.+\frac{k_{\perp}^{2}}{k_{\|}^{2}}\left[1+\frac{\beta_{j \perp}}{2 \beta_{j \|}} Z^{\prime}\left(\frac{\omega}{k_{\|}\left\langle v_{j \|}\right\rangle}\right)\right]\right\}
\end{aligned}
$$

The meaning of the symbols is conventional. $Z\left(\zeta_{j}\right)$ is the plasma dispersion function of argument $\zeta_{j}=\omega / k_{\|}\left\langle v_{j \|}\right\rangle$, and the prime indicates the derivative with respect to its argument. Furthermore, $v_{A}$ is the total Alfven velocity (summed over all species $j$ ), and $\left\langle v_{j \|}\right\rangle=\left(2 k_{B} T_{j \|} / m_{j}\right)^{1 / 2}$ is the average (thermal) parallel speed. The latter can also be expressed in terms of the component $j$ Alfvén speed

$$
\left\langle v_{j \mid !}\right\rangle^{2}=\beta_{j \perp} v_{j A}^{2} /\left(A_{j}+1\right)
$$

The general very low frequency $\left(|\omega| \ll \Omega_{i},|\omega| \ll\left|k_{\|}\right|\left\langle v_{\|}\right\rangle\right)$ dispersion relation derived from the above relation in the limit of $k v_{A} \gg|\omega|$ and for the cold electron-proton plasma is (Hasegawa, 1975)

$$
k_{\|}^{2}\left[1+\frac{\beta_{\perp} A}{2(A+1)}\right]-k_{\perp}^{2}\left[\left(\beta_{\perp} A-1\right)+\right.
$$

$$
\left.+i \sqrt{\pi \beta_{\perp}} \frac{\omega}{k_{\|} v_{A}}(A+1)^{\frac{3}{2}}\right]=0
$$

As mentioned above inclusion of a finite isotropic electron temperature would cause an additional electron pressure effect as shown by Pantellini and Schwartz (1995) and extended by Pokhotelov et al. (1999). The instability condition of the mirror mode in a cold electron multi-component warm and anisotropic ion plasma has been given by Tajiri, (1967) and Hasegawa (1969) (see also Hasegawa, 1975; Treumann and Baumjohann, 1997). In a pure cold electron-anisotropic proton plasma close to perpendicular propagation it assumes the familiar mirror criterion

$$
\beta_{\perp} A>1 .
$$

Otherwise, for given $A>0$ and oblique propagation one obtains a condition on the propagation angle $\tan \theta=k_{\|} / k_{\perp}$ as

$$
\tan ^{2} \theta \geq \frac{1+\beta_{\perp} A / 2(A+1)}{\beta_{\perp} A-1}
$$

Marginal instability is obtained for the equal sign in the above expression proving that the mirror wave is an oblique mode.

The mirror mode competes with the parallel electromagnetic ion cyclotron instability (Gary 1992,1993) which goes unstable under the condition that the resonant ions have positive anisotropy $A>0$ and energy larger than Alfvern energy $m_{i} v_{A}^{2} / 2$ which in the magnetosheath is the order of $100 \mathrm{eV}$. Its growth rate is proportional to the generally small fraction $\Delta n_{\ell \text { res }} / n_{0} \ll 1$ of resonant particles $v_{\ell_{\|}}=\mid\left(\omega-\Omega_{c \ell}\right) / k_{i \mid}$ satisfying these conditions. In addition, adding a few $\%$ of $\mathrm{He}^{++}$ions reduces the ion cyclotron growth rate due to additional to damping. As shown by Southwood and Kivelson (1993), the mirror mode is in contrast sensitive to the numerous $v_{\| 1} \sim 0$ particles. Hence, though the ion cyclotron waves may be unstable under similar conditions, the mirror mode may still grow faster in the magnetosheath.

\subsection{Spatial Amplification}

The observations of mirror modes in space plasmas and in particular in the magnetosheath (e.g. Tsurutani et al., 1982, 1984; Hubert et al., 1989; Lacombe et al., 1992; Fazakerley and Southwood, 1994a,b; Lacombe and Belmont, 1995; Czaykowska et al., 1998; Lucek et al., 1999a, and others) cannot distinguish between temporarily growing and spatially amplified nonlocal mirror modes. Some of the observed waves seem to exhibit spatially growing amplitudes when passing through the magnetosheath and approaching the magnetopause It is well known that such mirror modes are convected with the plasma flow. Two-spacecraft observations are required in order to infer about the local or convective character of the modes. In the absence of any clear distinction between both cases it is therefore of interest to investigate the convective mirror mode.

As is transparent from the above derivation of the temporal locally growing mirror mode, linear mirror mode analysis yields a purely imaginary frequency $\omega=i \gamma$ given, for 
instance, in Hasegawa (1975). Generalization of the mirror instability to a plasma streaming with velocity $\mathrm{V}$ is trivially done by replacing $\omega \rightarrow \omega-\mathbf{k} \cdot \mathbf{V}$. This attributes a real part to the frequency yielding

$$
\omega=\mathbf{k} \cdot \mathbf{V}+i \gamma
$$

The derivation of the dispersion relation (1) is, however, valid for general real or complex wave numbers. Suppose that $\omega$ is real while $\mathbf{k}$ is a complex vector. We define, $a=$ $1+\frac{1}{2} \beta_{\perp} A /(A+1), \epsilon=\left(\beta_{\perp} A-1\right) / a>0$ and $b^{2}=$ $\left(\pi \beta_{\perp}\right)(A+1)^{3} / a^{2}$. Taking $\omega$ and $k_{\perp}$ real and setting $k_{\|}=$ $K_{\|}-i \Gamma_{\|}$, with positive $\Gamma_{\|}$corresponding to spatially amplified disturbances the dispersion relation (4) can be split into real and imaginary parts. This leads to the following two equations

$$
\begin{aligned}
K_{\|}^{2} & =3 \Gamma_{\|}^{2}+k_{\perp}^{2} \epsilon \\
\Gamma_{\|}\left(3 K_{\|}^{2}-\Gamma_{\|}^{2}\right) & =k_{\perp}^{2}\left(\epsilon \Gamma_{\|}-\frac{b \omega}{v_{A}}\right)
\end{aligned}
$$

In the low frequency parameter domain these equations can be shown to have no solution for the wave numbers of interest, $K_{\|} \ll k_{\perp}$. The only solution is one where $K_{\|}>\sqrt{\epsilon} k_{\perp}$, and this is amplified (evanescent) along the field for $\omega<0$ $(\omega>0)$.

Let us consider perpendicular amplification. We assume that $k_{\perp}=K_{\perp}-i \Gamma_{\perp}$ is complex and $k_{\|}, \omega$ are both real. The real and imaginary parts of the dispersion relation (4) yield the following expression for the perpendicular amplification rate

$$
\Gamma_{\perp}^{2}=K_{\perp}^{2}-Q k_{\sharp}^{2}
$$

where $Q=\epsilon /\left(\epsilon^{2}+b^{2} \omega^{2} / k_{\|}^{2} v_{A}^{2}\right)$ is a positive quantity. For $\epsilon=0$ conditions the perpendicular amplification length is the same as the perpendicular scale of the mirror mode, while for $\epsilon>0$ one has $\Gamma_{\perp}<K_{\perp}$. Clearly, this expression limits the perpendicular wavenumber from below:

$$
\frac{K_{\perp}^{2}}{k_{\|}^{2}}>\frac{\epsilon}{\epsilon^{2}+\left(b \omega / k_{\|} v_{A}\right)^{2}}
$$

This estimate is valid for $\epsilon>0$. At $\epsilon=0$ there is no restriction on $K_{\perp}$. The general equation for $K_{\perp}$ is a bi-quadratic equation

$$
K_{\perp}^{4}-a_{1} K_{\perp}^{2}-a_{2}=0
$$

where

$$
a_{1}=Q k_{\|}^{2}, \quad a_{2}=\frac{k_{\|}^{4} Q^{2} b^{2}}{4 \epsilon^{2}} \frac{\omega^{2}}{k_{\|}^{2} v_{A}^{2}}
$$

The only one real solution is

$$
K_{\perp}^{2}=\frac{Q k_{\|}^{2}}{2}\left\{1+\left(1+\frac{b^{2}}{\epsilon^{2}} \frac{\omega^{2}}{k_{\|}^{2} v_{A}^{2}}\right)^{\frac{1}{2}}\right\}
$$

It yields a perpendicular amplification rate

$$
\Gamma_{\perp}^{2}=\frac{k_{\|}^{2} Q}{2}\left\{\left[1+\frac{b^{2} \omega^{2}}{\epsilon^{2} k_{\|}^{2} v_{A}}\right]^{\frac{1}{2}}-1\right\}
$$

Since this is always positive, one of the modes is amplified in the perpendicular direction. In the very low frequency limit $\omega \ll k_{\sharp} v_{A} \epsilon / b$ one has $Q \approx \epsilon^{-1}$, and the amplification rate is just

$$
\Gamma_{\perp} v_{A} \simeq b\left(\omega / 2 \epsilon^{3}\right)^{\frac{1}{2}}, \quad \omega \ll k_{\|} v_{A}
$$

In this case the parallel phase velocity of the mode is small against the Alfvén speed and the waves are relatively short parallel wave length but longer than the ion inertial length, $\lambda \gg \lambda_{i} \equiv c / \omega_{p i}$, since $\omega \ll \Omega_{i}$. The mirror mode propagates slower than Alfvén speed along the field and has perpendicular wave number

$$
K_{\perp} \simeq k_{\|} / \epsilon^{\frac{1}{2}}
$$

This must be large compared to the parallel wave number. So $\epsilon$ must be small. These approximations hold close to marginal conditions. In the opposite case one has

$$
\Gamma_{\perp} \simeq K_{\perp}\left(\frac{k_{\|} v_{A}}{2 b \omega}\right)^{\frac{1}{2}} k_{\|}, \quad \omega \gg k_{\|} v_{A}
$$

which is practically independent of $\epsilon$. This case applies to very long parallel wave lengths, $\lambda \gg \lambda_{i}$. Mirror modes of this kind form cigars extended along the field. Finally it is worth mentioning that no mode can propagate for $k_{\| l}=0$.

\subsection{Convective Growth Rate}

The local growth rate for the above slowly oscillating modes can be found when observing the relation between the spatial amplification rate and the local temporal growth rate

$$
\gamma_{\perp}=-v_{g \perp} \Gamma_{\perp}
$$

which we have written here only for the perpendicularly amplified mode. The group velocity $v_{g \perp}=\partial \omega / \partial k_{\perp}$ can be calculated from the equation (12). This equation is conveniently written as

$$
K_{\perp}=\frac{k_{\|}}{\sqrt{2}}\left(\frac{Q}{\epsilon}\right)^{\frac{1}{4}}(1+\sqrt{\epsilon Q})^{\frac{1}{2}}
$$

In analogy the amplification rate assumes the form

$$
\Gamma_{\perp}=\frac{k_{\|}}{\sqrt{2}}\left(\frac{Q}{\epsilon}\right)^{\frac{1}{4}}(1-\sqrt{\epsilon Q})^{\frac{1}{2}}
$$

Using these expressions for the wave number and the amplification rate one obtains

$$
\gamma_{\perp}=\frac{\sqrt{2}}{b}\left(\frac{\epsilon}{Q^{5}}\right)^{\frac{1}{4}}(1+1 / 2 \sqrt{\epsilon Q})^{-1}
$$


Since this is given in terms of $Q$ which is a function of $\omega$ one can also express it solely through the perpendicular wavenumber

$$
\frac{\gamma_{\perp}}{K_{\perp} v_{A}}=\frac{\epsilon^{\frac{3}{2}}}{4 b} \frac{C}{(C-1)^{\frac{3}{2}}}
$$

where $C \equiv\left(1+8 \epsilon K_{\perp}^{2} / k_{\|}^{2}\right)^{\frac{1}{2}}$. This is a complicated expression. It simplifies whenever $K_{\perp}$ is sufficiently large compared with $k_{\|}$so that it is justified to neglect the one in the expression for $C$. Then the nonlocal growth rate which is related to the amplification rate becomes

$$
\gamma_{\perp} \approx \frac{v_{A} \sqrt{K_{\perp} k_{\|}}}{4 b}\left(\frac{\epsilon^{5}}{8}\right)^{\frac{1}{4}}
$$

For the physical understanding one may note that the perpendicular group velocity in (17) is negative for the growing mode. This fact implies that energy is flowing into the mode along the perpendicular direction and out of the mode along the magnetic field. This perpendicular inflow is another expression for the inflation of the mirror bubbles experienced during amplification.

\section{Mirror Mode Trapping}

Under conditions as those near the magnetopause the mirror mode has been observed to reach large amplitudes and being concentrated mainly close to the magnetopause (Hubert et al., 1989; Hill et al., 1995; Lacombe and Belmont, 1995; Lucek et al., 1999a,b). Similarly, short wavelength mirror modes hasve been identified close to the bow shock (Lacombe et al., 1992; Czaykowska et al., 1998). We here investigate another possibility which relates to the gross inhomogeneity of plasma conditions near the magnetopause and bow shock. Since the mirror mode is little affected by density perturbations we consider the case of mirror modes being captured between two magnetic walls.

Let the width of the magnetic trough between the walls be $L$. We assume the walls are standing discontinuities one of them the magnetopause the other a hypothetical discontinuity situated inside the magnetosheath. This could of course be served by the bow shock itself, however in most cases it is too far away from the magnetopause in order to affect the evolution of mirror modes. Thus the latter requires a more lengthy discussion as there are no really good theoretical reasons for assuming discontinuities to exist in the magnetosheath. Observations have sometimes shown (cf. Hill et al., 1995) though that the nature of the turbulence in the magnetosheath abruptly changes character when approaching the magnetopause. Steepened standing mhd wave like a slow mode wave or and Alfvén wave could cause temporary and convecting discontinuities in the sheath. On the other hand closer to the magnetopause and for Petschek reconnection one would expect standing slow mode shocks to exist at a narrow opening angle in front of the magnetopause. In this case the following discussion applies to the narrow region between these shocks and the magnetopause. Hence, in application to the sheath there is no satisfactory justification of such a second boundary. We nevertheless investigate what will happen to mirror modes inc ase such a magnetic wall existed.

The pressure balance condition $p_{\perp}+B^{2} / 2 \mu_{0}=$ const holding between the plasma and magnetic fields inside and outside the two magnetic walls implies that $\beta$ will be high enough for the mirror mode to evolve only in the inside region. We consider the case of perpendicular amplification. Trapping of the mirror mode between the magnetic walls implies that the perpendicular wave number of the trapped modes vanishes at the two discontinuities at $x= \pm L / 2$. Hence the existence of the walls will select a certain discrete spectrum of mirror modes which can exist inside the trough. In order to determine the spectrum of mirror wave modes we can apply the quantization condition

$$
\int_{-L / 2}^{L / 2} K_{\perp}(x) \mathrm{d} x=\left(\ell+\frac{1}{2}\right) \pi
$$

where $\ell=0,1, \ldots$ is the quantum number, and $x$ is the transverse spatial coordinate. For a rectangular trough $B=B_{1}>$ $B_{0}$ for $x<-L / 2, B=B_{0}$ for $-L / 2 \leq x \leq L / 2$, and $B=B_{2}>B_{0}$ for $x>L / 2$, and $B_{0}=$ const $<B_{1}, B_{2}$, the wave number $K_{\perp}$ does not depend on $x$, and we obtain

$$
L K_{\perp}=(\ell+1 / 2) \pi
$$

which shows that the perpendicular wavelengths follow a $\lambda_{\perp \ell} \propto 2 L(\ell+1 / 2)^{-1}$ dependence. After substitution from (12) for $K_{\perp}$ into the above equation the following condition is obtained:

$$
\sqrt{\epsilon Q}(1+\sqrt{\epsilon Q})=\frac{2 \pi \epsilon}{4 k_{\| !}^{2} L^{2}}\left(\ell+\frac{1}{2}\right)^{2}
$$

which is a quadratic equation for $\sqrt{\epsilon Q}$. The only physical reasonable solution for $\epsilon Q$ is

$$
4 \epsilon Q=\left[(1+4 q)^{2}-1\right]^{2}
$$

where $q$ is the right-hand side of equation (25). Solving for the frequency yields the following expression:

$$
\left(\frac{b \omega}{\epsilon k_{\|} v_{A}}\right)^{2}=\frac{4-[\sqrt{1+4 q}-1]^{\frac{1}{2}}}{[\sqrt{1+4 q}-1]^{\frac{1}{2}}}
$$

For the numerator to be positive the parallel wavelengths have to satisfy the inequality

$$
k_{\|} L>\frac{(\ell+1 / 2) \epsilon^{\frac{1}{2}}}{2 \sqrt{112}}=0.05 \epsilon^{\frac{1}{2}}\left(\ell+\frac{1}{2}\right)
$$

Actually, this is a condition either on the quantum number $\ell$ or on the parallel wavelength. For the latter it requires

$$
\lambda_{\|} \leq \frac{40 \pi L}{\epsilon^{\frac{1}{2}}(\ell+1 / 2)}=20 \pi \lambda_{\perp} \epsilon^{-\frac{1}{2}}
$$


The allowed range of parallel wavelengths thus exceeds the perpendicular wavelength $\lambda_{\perp}=2 L /(\ell+1 / 2)$ by roughly a factor of $60 / \sqrt{\epsilon}$. For the equal sign in the above conditions the frequency is exactly zero. In the case of small $q$ the frequency follows the quantization law

$$
\frac{\omega_{\ell}}{k_{\| v_{A}}} \approx \frac{2 \epsilon}{b(2 q)^{\frac{2}{4}}}=\frac{2}{b}\left(\frac{\epsilon^{3}}{\pi}\right)^{\frac{1}{4}}\left(\frac{k_{\|} L}{\ell+1 / 2}\right)^{\frac{1}{2}}
$$

The frequency spacing of two adjacent quantum harmonics thus follows the nonlinear law

$$
\Delta_{\ell}=\omega_{\ell+1}^{2}-\omega_{\ell}^{2}=-(\ell+3 / 2)^{-1} \omega_{\ell}^{2}
$$

It is negative implying that the frequencies in the spectrum decrease with increasing $\ell$. Moreover, the spacing between the frequencies also decreases. The highest possible frequency trapped in the slab yields the first order spacing

$$
\Delta_{0}=-1.5 \omega_{0}^{2} .
$$

with

$$
\omega_{0}=\frac{2}{b}\left(\frac{\epsilon^{3}}{\pi}\right)^{\frac{1}{4}} \sqrt{2 k_{\|} L}, \quad K_{\perp 0}=\frac{\pi}{2} L
$$

Because $k_{\|} L$ is limited this equation implies that the zero order frequency must be larger than

$$
\omega_{0} \geq \frac{2}{5 \pi^{\frac{1}{4}}} \frac{\epsilon^{2}}{b}
$$

which gives an estimate for the lowest order frequency spacing of the trapped modes:

$$
\left|\Delta_{0}\right| \geq \frac{0.24}{\sqrt{\pi}} \frac{\epsilon^{4}}{b^{2}}
$$

Though it is rather simplistic, the current model provides some basic insight into the trapping of mirror modes. It assumes that the mirror wave is trapped in a rectangular magnetic trough with two tangential discontinuities representing its boundaries. A more realistic model may assume a Gaussian profile of the magnetic field $B / B_{0}=\exp \left(x^{2} / L^{2}\right)$. In this case the wave is reflected at the positions $x= \pm x_{0}$ where the perpendicular wavenumber vanishes. It can be shown that this happens first for $x_{0}^{2}=(L / 2) \ln \left(1 / A \beta_{\perp 0}\right)$ where $\beta_{\perp 0}=$ $\beta_{\perp}\left(B_{0}\right)$. Solution of the resulting complicated quantization integral can be performed only numerically and will be given elsewhere.

\section{Discussion}

Trapping of mirror modes in a magnetic trough is an interesting case. Since mirror modes need high $\beta$ conditions, mirror waves will be trapped inside magnetic boundaries of strong magnetic field. The magnetopause under tangential discontinuity conditions may act as one such boundary. Clearly, $\beta$ generally drops to low values when crossing the magnetopause from the magnetosheath into the magnetosphere. Mirror modes will therefore be heavily suppressed inside the magnetopause and will be reflected when encountering the magnetopause. In modeling a magnetic trap, the bow shock may act as another external boundary under conditions when the magnetosheath is sufficiently compressed due to high solar wind pressure conditions. This may not happen very frequently but when it happens trapping of long perpendicular wave length mirror modes should become possible.

Some kind of boundaries and changes in the character of sheath waves have also been found in the magnetosheath region adjacent to the magnetopause. Song et al. (1992a, b) have interpreted these structures as slow shocks. Though in the Petschek model of reconnection at the magnetopause slow shocks should naturally appear, there is still much disagreement about their observational reality. The magnetic field is known to decrease across slow shock waves which would provide conditions for mirror mode trapping. Observations sometimes also indicate changes in the plasma parameters and magnetic field even deeper inside the sheath though still far away from the magnetopause in relation to the observation of mirror modes (e.g. Hill et al., 1995; Lucek et al., $1999 \mathrm{a}, \mathrm{b})$. However, these observations are all to vague in order to being taken as evidence for magnetosheath mirror mode trapping of the kind argued for in the present paper.

A more conincing possibility for mirror mode trapping arises in the external cusp region where the high- $\beta$ anisotropic pressure magnetosheath plasma is confined between the equatorward and poleward edges of the cusp provided by the magnetopause. Whithin the slab model developed in this communication one can understand the observation of cusp mirror waves as modes trapped between these boundaries. We have shown that only particular wavelengths can exist inside such a trap. The slab selects the possible modes and may be responsible for the appearance of only a few well distinguishable wavelengths in observations of mirror modes.

A slab model is of course not the best approximation to real conditions. However, in view of the difficulty of solving a more realistic Gaussian model for the spatial dependence of the magnetic field, such a simplistic model may give insight into the trapping physics. The interesting conclusion is that the spacing in wavelength space of the few trapped modes inhibits a broad spectrum of mirror modes to evolve.

Trapping of mirror modes leads to the formation of standing mirror bubbles. It supports the mirror mode to quickly reach a nonlinear state that is characterized by large amplitude variations in the magnitude of the magnetic field and the splitting of the particle distribution function inside the bubbles into two components, a trapped component and a passing component. Quasilinear theory (e.g. Shapiro and Shevchenko, 1964) is unable to describe this phenomenon. Very promising nonlinear approaches have been presented by Kivelson and Southwood (1996) and Pantellini (1998).

Acknowledgements. Part of this work has been performed under a senior visiting scientist program at ISSI. R.A.T. thanks the directors of ISSI, J. Geiss, G. Paschmann, and R. von Steiger, for their continuous support during the course of this work. He also thanks the ISSI staff for their hospitality, Another part of this research resulted in the context of an AMPTE IRM-UKS Team work organized at ISSI. The authors thank M. Balikhin, M. Dunlop, A. 
Fazakerley, G. Paschmann and S. Schwartz for their contributions and stimulating discussions. They also thank the two referees for their constructive comments. We note that our colleague Norbert Sckopke, who participated in the early work on this subject, died unexpectedly on November $28,1999$.

\section{References}

Barnes, A. Collisionless damping of hydromagnetic waves, Phys. Fluids, 9, $1483,1966$.

Baumjohann, W., Treumann, R. A., Georgescu, E., Haerendel, G., Fornacon, K.-H., and Auster, U., Ann. Geophysicae, 17, 1528, 1999.

Chandrasekhar, S., Kaufman, A. N., and Watson, K. M., The stability of the pinch, Proc. R. Soc. London, A 245, 435, 1958.

Czaykowska. A., Bauer, T. H., Treumann, R. A., Baumjohann, W., Mirror waves downstream of the quasi-perpendicular bow shock, J. geophys. Res., 103, 4747, 1998.

Fazakeriey, A. N., and Southwood, D. J., Mirror instability in the magnetosheath, Adv. Space Res., 14, 65, 1994a.

Fazakerley, A. N., and Southwood, D. J., Theory and observation of magnetosheath waves, in: Solar Wind Sources of Magnetospheric Ultra-LowFrequency Waves, Geophys. Monogr. Ser., Vol. 81, M. J. Engebretson, K. Takahashi, and M. Scholer (eds.), p. 147, AGU, Washington, D.C., $1994 \mathrm{~b}$.

Fazakerley, A. N., Coates, A. J., and Dunlop, M. W., AMPTE-UKS observations of ion velocity distributions associated with magnetosheath waves, Adv. Space Res., 15, 349, 1995.

Gary, S. P., The mirror and ion cyclotron anisotropy instabilities, J. geophys. Res., 97, 8523, 1992.

Gary, S. P., Theory of Space Plasma Microinstabilities, Cambridge University Press, Cambridge, 1993.

Glassmeier, K.-H., Motschmann, U., Mazelle, C., Neubauer, F. M., Sauer, K., Fuselier, S. A., and Acuña, M. N., Mirror modes and fast magnetosonic waves near the magnetic pile-up boundary of comet P/Halley, $J$. geophys. Res., 98, 20955, 1993.

Hasegawa, A., Drift-mirror instability in the magnetosphere, Phys. Fluids, $12,2642,1969$.

Hasegawa, A., Plasma Instabilities and Nonlinear Effects, p. 94, Springer Verlag, Heidelberg, 1975.

Hill, P., Paschmann, G., Treumann, R. A., Baumjohann, W., and Sckopke, N., Plasma and magnetic field behavior across the magnetosheath near local noon, J. geophys. Res., 100, 9575, 1995.

Hollweg, J., and Volk, H., New plasma instabilities in the solar wind, J. geophys. Res., 75, 5297, 1970.

Hollweg, J., and Volk, H., Energy and momentum exchange in transverse plasma waves, J. geophys. Res., 76, 7527, 1971.

Hubert, D., Perche, C., Harvey, C.C., Lacombe, C., and Russell, C. T., Observation of mirror waves downstream of a quasi-perpendicular shock, Geophys. Res. Lett., 16, 159, 1989.

Kivelson, M. G., and Southwood, D. J., Mirror instability II: The mechanism of nonlinear saturation, J. geophys. Res., 101, 17365, 1996.

Lacombe, C., and Belmont, G., Waves in the Earth's magnetosheath: Observations and interpretations, Adv. Space Res., 15, 329, 1995.

Lacombe, C. F., Pantellini, G. E., Hubert, D., Harvey, C. c., Mangeney, A.,
Belmont, G., and Russell, C. T., Mirror and Alfvénic waves observed by ISEE 1-2 during crossings of the Earth's bow shock, Ann. Geophys., 10, $772,1992$.

Lucek, E. A., Dunlop, M. W., Balogh, A., Cargill, P., Baumjohann, W., Georgescu, E., Haerendel, G., and Fornacon, K.-H., Mirror mode structures observed in the dawnside magnetosheath by Equator-S, Geophys. Res. Lett., 26, 2159, 1999a.

Lucek, E. A., Dunlop; M. W., Balogh, A., Cargill, P., Baumjohann, W., Georgescu, E., Haerendel, G., and Fornacon, K.-H., Identification of magnetosheath mirror modes in Equator-S magnetic field data, Ann. Geophys., 17, 1560, 1999b.

Pantellini, F. G. E., and S. J. Schwartz, Electron temperature effects in the linear proton mirror instability, J. geophys. Res., 100, 3539, 1995.

Pantellini, F. G. E., A model of the formation of stable nonpropagating magnetic structures in the solar wind based on the nonlinear mirror instability, J. geophys. Res., 103, 4789, 1998.

Pokhotelov, O. A., Balikhin, M. A., Alleyne, H. St.-C., and Onishchenko, O. G., Mirror instability with finite electron temperature effects, $J$. geophys. Res., 105, 2393, 2000.

Price, C. P., Switt, D. W., and Lee, L. C., Numerical simulations of nonoscillatory mirror waves in the Earth's magnetosheath, J. geophys. Res., 91, $101,1986$.

Rudakov, L. I., and Sagdeev, R. Z., On the instability of a nonuniform rarefied plasma in strong magnetic field, Dokl. Akad. Nauk SSSR, Engl. Transl., 6, 415, 1961.

Schwartz, S. J., Burgess, D., and Moses, J. J., Low-frequency waves in the Earth's magnetosheath: present status, Ann. Geophysicue, 14, 1134, 1996.

Shapiro, V. D., and Shevchenko, V. I., Quasilinear theory of instability of a plasma with an anisotropic ion velocity distribution, Sov. Phys. JETP, $18,1109,1964$

Song, P., Russell, C. T., and Thomsen, M. F., Slow mode transition in the frontside magnetosheath, $J$. geophys. Res., 97, 8295, 1992a.

Song, P., Russell, C. T., and Thomsen, M. F., Waves in the inner magnetosheath: A case study, Geophys. Res. Lett, 9, 2191, 1992a.

Southwood, D. J., and Kivelson, M. G., Mirror instability I: The physical mechanism of linear instability, J. geophys. Res., 98, 9181, 1993.

Tajiri, M., Propagation of hydromagnetic waves in coltisionless plasma, II, Kinetic approach, J. Phys. Soc. Jpn., 22, 1482, 1967.

Tátrallyay, M., and Erdös, G., Investigation of mirror mode structures using multiple spacecraft measurements, Proceedings of an ESA conference on Cluster $I I$, in press, 2000.

Treumann, R. A., and Baumjohann, W., Advanced Space Plasma Physics, Imperial College Press, London, 1997.

Tsurutani, B. T., Smith, E. J., Anderson, R. R., Ogilvie, K. W., Scudder, J, D., Baker, D. N., and Bame, S. J., Lion roars and nonoscillatory drift mirror waves in the magnetosheath, J. geophys. Res., 87, 6060, 1982.

Tsurutani, B. T., Richardson, I. G., Lepping, R. P., Zwickl, R. D., Jones, D. E., Smith, E. J., and Bame, S. J., Drift mirror mode waves in the distant ( $X \simeq 200 \mathrm{R}_{\mathrm{E}}$ ) magnetosheath, Geophys, Res. Lett., II, $1102,1984$.

Tsurutani, B. T., Southwood, D. J., Smith, E. J., and Bajogh, A., Nonlinear magnetosonic waves and mirror mode structures in the March 1991 Ulysses interplanetary event, Geophys. Res. Lett., 19, 1267, 1992. 